\title{
MEMS Accelerometer with Screen Printed Piezoelectric Thick Film
}

\author{
Hindrichsen, Christian Carstensen; Lau-Moeller, R.; Bove, T.; Thomsen, Erik Vilain
}

Published in:

Proceedings of the IEEE Sensors, IEEE, 2006

Link to article, DOI:

10.1109/ICSENS.2007.355913

Publication date:

2007

Document Version

Publisher's PDF, also known as Version of record

Link back to DTU Orbit

Citation (APA):

Hindrichsen, C. C., Lau-Moeller, R., Bove, T., \& Thomsen, E. V. (2007). MEMS Accelerometer with Screen Printed Piezoelectric Thick Film. In Proceedings of the IEEE Sensors, IEEE, 2006 IEEE. https://doi.org/10.1109/ICSENS.2007.355913

\section{General rights}

Copyright and moral rights for the publications made accessible in the public portal are retained by the authors and/or other copyright owners and it is a condition of accessing publications that users recognise and abide by the legal requirements associated with these rights.

- Users may download and print one copy of any publication from the public portal for the purpose of private study or research.

- You may not further distribute the material or use it for any profit-making activity or commercial gain

- You may freely distribute the URL identifying the publication in the public portal 


\section{MEMS Accelerometer with Screen Printed Piezoelectric Thick Film}

\author{
C. C. Hindrichsen and E. V. Thomsen \\ Department of Micro and \\ Nanotechnology (MIC) \\ Technical University of Denmark (DTU) \\ Lyngby, Denmark \\ cch@mic.dtu.dk
}

\author{
R. Lou-Møller \\ Ferroperm Piezoceramics A/S \\ Kvistgaard, Denmark
}

\author{
T. Bove \\ Insensor $\mathrm{A} / \mathrm{S}$ \\ Kvistgaard, Denmark
}

\begin{abstract}
A bulk-micromachined piezoelectric MEMS accelerometer with screen printed piezoelectric $\mathrm{Pb}\left(\mathrm{Zr}_{\mathrm{x}} \mathrm{Ti}_{1-\mathrm{x}}\right) \mathrm{O}_{3}$ (PZT) thick film (TF) as the sensing material has been fabricated and characterized. The accelerometer has a four beam structure with a central seismic mass $(3600 \times 3600 \times 500$ $\mu \mathrm{m} 3)$ and a total chip size of $10 \times 10 \mathrm{~mm} 2$. The resonance frequency is found to be $17.5 \mathrm{kHz}$ and the sensitivity $4.15 \mathrm{mV} / \mathrm{g}$ for a single beam.
\end{abstract}

\section{INTRODUCTION}

Piezoelectric materials as PZT have the ability to convert mechanical forces to an electrical signal and vice versa. Screen printed TF PZT integrated with MEMS technology is a way to get smaller, more integrated and cheaper sensors with increased functionality. The high coupling between mechanical and electrical energy is well suited for state-ofthe-art accelerometers sensing dynamic accelerations in a high bandwidth.

A MEMS uniaxial accelerometer with TF PZT has been presented by Wang [1] with a $7 \mu \mathrm{m}$ thick PZT element and a sensitivity of up to $7.86 \mathrm{mV} / \mathrm{g}$. The design in [1] consists of an anular membrane and can not be used for tri axial measurements. In [2] an accelerometer with four beams and a central seismic mass is presented but with a low sensitivity of $0.1 \mathrm{mV} / \mathrm{g}$ and a resonance frequency of $7.55 \mathrm{kHz}$.

The sensitivity is proportional to the thickness of the PZT element. The design and process flow presented here is optimized for uni-axial accelerations but can be used for the development of a tri-axial accelerometer. The thickness of the PZT element is $60 \mu \mathrm{m}$ allowing higher sensitivities than possible with thin film technology. A four beam structure allows for measurements of rotations of the seismic mass due to in-plane accelerations.

\section{DESIGN}

Silicon is used as the substrate material due to its mechanical properties and the advanced fabrication processes available today. The accelerometer consist of a

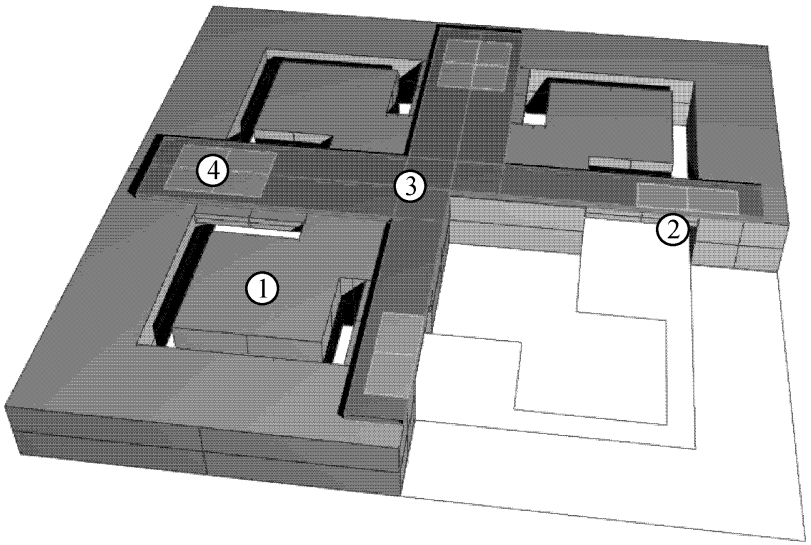

Figure 1. Concept drawing of the accelerometer with one central seismic mass (1) and four beams (2) with TF PZT (3) and Au top electrodes (4) on. The bottom electrode is covering the whole surface. Note that one fourth of the accelerometer is removed.

central seismic mass (1), $\sim 16 \mathrm{mg}$, fixed by four beams (2) and with the sensing PZT layer (3) screen printed on top of each beam as shown in Fig. 1. Vertical acceleration will cause a deflection of the seismic mass and bend the four beams in a S-shape as shown in Fig. 2. The bending will induce an electrical field between the top (4) and the bottom electrode covering the whole surface. Each beam will give the same output signal at vertical accelerations. In-plane acceleration causing a rotation of the seismic mass will give inverse output signals for two opposite beams. The design is optimized for vertical acceleration but the concept with four beams allows for tri-axial acceleration measurements in future designs. FEM modeling is used to optimize the dimensions of the design in order to have a high enough resonance frequency.

The resonance frequency and the sensitivity are determined by the dimensions of the four supporting beams and the size of the seismic mass. The in-plane shape of the seismic mass is optimized in order to occupy as much area 


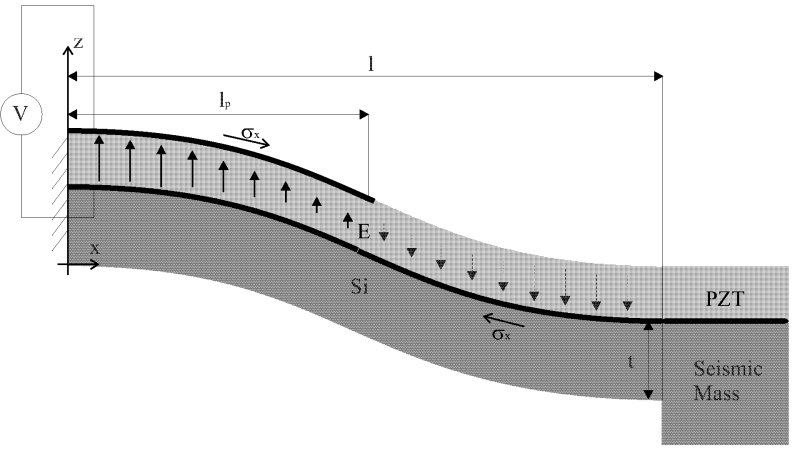

Figure 2. The S-shape of the deflection will cause a non uniform E field in the PZT layer. The overlap of the top and bottom electrode, $1_{p}$, should not be longer than half the beam length, 1 . The E field on the right half of the beam would only occur if there were an top electrode on the right half of the beam .

as possible. FEM modeling is used to optimize the dimensions of the design.

To maximize the sensitivity, it is vital that the active PZT layer does not extend all the way out to the seismic mass, since there will be a negative stress contribution, $\sigma_{\mathrm{x}}$, and opposite electric field, E, close to the seismic mass. Fig. 2 shows the shape of the deflection and the E field in the PZT layer. Only the part of the PZT layer between the top and bottom electrode will be the active part of the PZT. The length of the top electrode, $1_{p}$, is half the length of the beam, 1.

The ratio between the thickness of the PZT layer and the silicon beam is optimized in order to have the neutral plane just in between the two layers. The neutral plane coordinate, $\underline{z}$, is determined by the geometry of the PZT layer and the silicon beam, as the ratio of the widths, $\alpha$, the ratio of Young's modulus for PZT and silicon, $r$, and the ratio of the thicknesses, $\beta$.

$$
\underline{\mathrm{z}}=\frac{\frac{1}{2}+\mathrm{r} \alpha \beta\left(\frac{1}{2} \beta+1\right)}{1+\mathrm{r} \alpha \beta} \mathrm{t}
$$

Limited resolution in the screen printing technique sets an upper limit for, $\beta$. All dimensions of the accelerometer are listed in Table 1.

TABLE I. The most important dimensions of the accelerometer.

\begin{tabular}{|c|c|}
\hline \multicolumn{2}{|c|}{ Dimensions $[\boldsymbol{\mu} \mathbf{~ m}]$} \\
\hline Width of Si beams & 1200 \\
\hline Width of PZT layer & 800 \\
\hline Length of Si beams $(\mathrm{l})$ & 800 \\
\hline Length of top electrode on beam $\left(\mathrm{l}_{\mathrm{p}}\right)$ & 300 \\
\hline Width of seismic mass & 3600 \\
\hline Thickness of Si beam & 37 \\
\hline Thickness of PZT layer & 60 \\
\hline
\end{tabular}

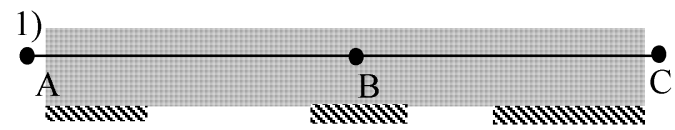

2)

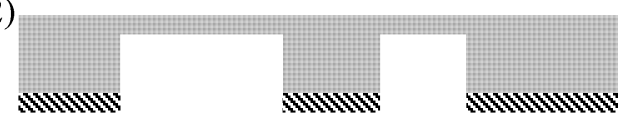

3)

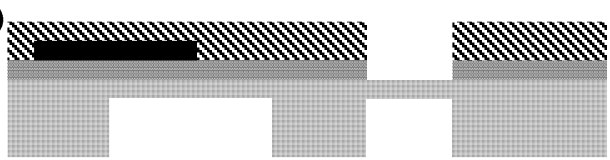

4)

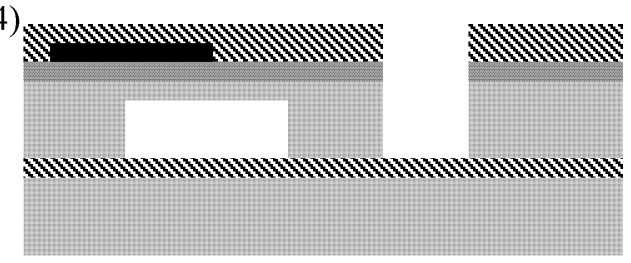

5)
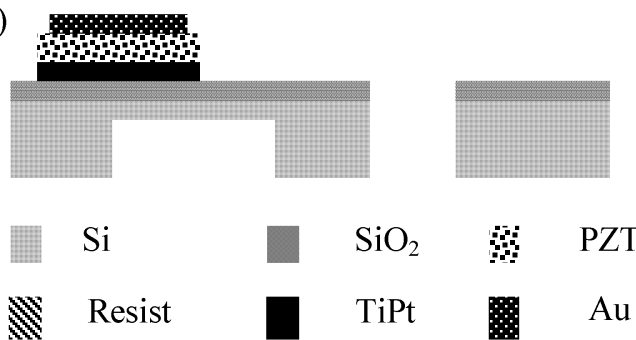

Figure 3. The process flow for the accelerometer uses three photo lithographic masks and two masks for the screen printing process. A ASE process is used to etch out the seismic mass and the four beams.

\section{FABRICATION}

The accelerometer is fabricated with standard MEMS processing and for the TF PZT layer a screen printing technique is used. The clean room process flow (see Fig. 3) has been developed, aiming to eliminate post processing

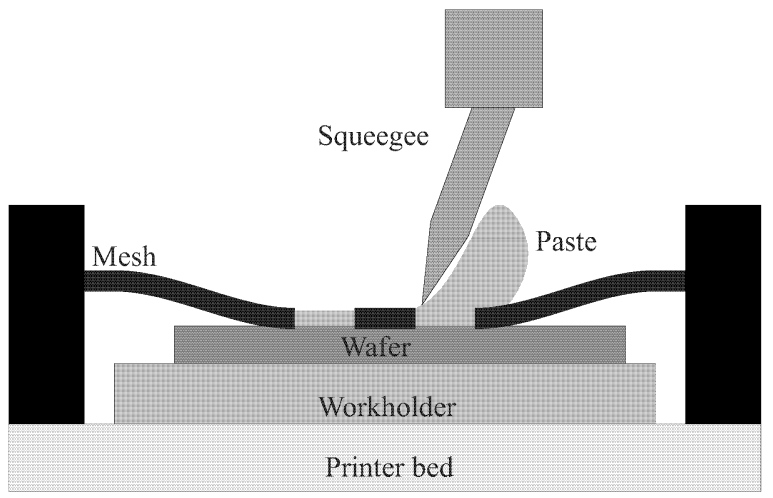

Figure 4. Shematic drawing of the screen printing process. A thick PZT paste is squeezed through a metal mask patterning the PZT elements. The same process is used for the Au top electrode. 


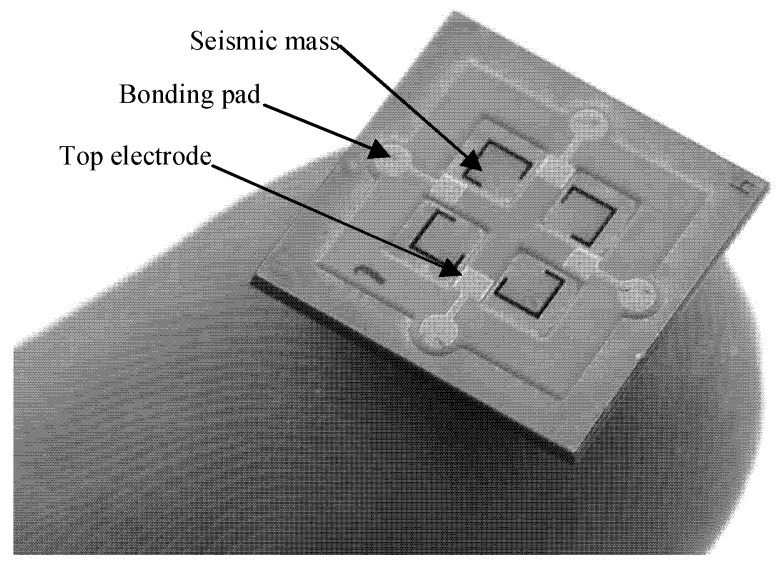

Figure 5. Accelerometer with the central seismic mass and the four beams on a finger tip. Signal read out is done with wire bonding connected to the four circular bonding pads.

after the TF deposition. $500 \mu \mathrm{m}$ thick double-side polished (100) 4" silicon wafers are used as substrate material. Three photolithographic masks and two additional masks for the screen printing process are used in the fabrication process.

An Advanced Silicon Etch (ASE) process is used for thinning down the beams from the backside to get the desired thickness of the silicon beams, $t$ (step 1-2). The beam thickness, $t$, is set by controlling the etch time. Standard positive photo resist is used as the masking material. A dedicated diffusion barrier layer and the bottom electrode layers are electron beam deposited on the front side. $|\mathrm{Ti}| \mathrm{Pt}|\mathrm{SiO} 2|$ has proven to be good stack of barrier layers [2]. The metal layer stack is covering the whole wafer except where the second ASE process is utilized from the front side. A lift off process of the negative photo resist is used to define the pattern of the metal layers (step 3). Mask 2 is used in a negative lithographic process and mask 3 is the inverse of mask 2 and is used in a positive lithographic process. For releasing the seismic mass and the beams the second ASE process is used from the front side (step 4). A

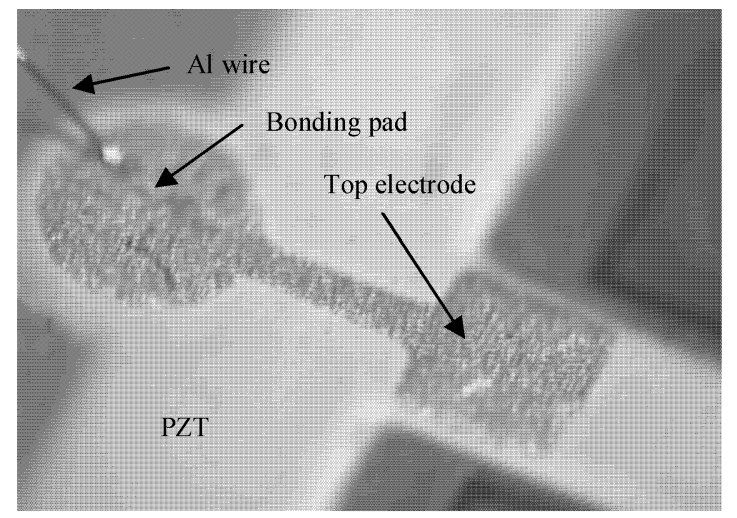

Figure 6. The Au top electrode $(5 \mu \mathrm{m})$ on the PZT layer $(60 \mu \mathrm{m})$. The black areas are the pattern from the ASE through-etch. A thin Al wire is attached to the bonding pad signal read out.

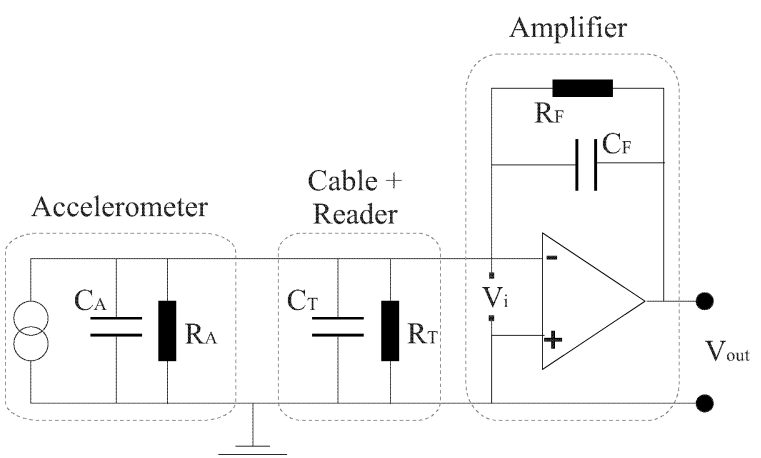

Figure 7. A charge amplifier is used to transform the high impedance signal from the accelerometer into a low impedance signal. Cable length and input impedance of the read out equipment is not affecting the signal

handle wafer is bonded to the backside enabling a throughetch of the wafer. Standard positive photo resist is used as masking material.

Following the conventional MEMS processing, a $60 \mu \mathrm{m}$ TF PZT (INSENSOR TF2100) and the $5 \mu \mathrm{m}$ Au top electrode are screen printed through metal masks on the front side by InSensor A/S. During the screen printing process a certain pressure is applied onto the thin silicon beams. It proves possible to successfully screen print on silicon beams with thicknesses down to $20 \mu \mathrm{m}$ without breaking them. Fig. 4 illustrates the screen printing process.

The final accelerometer is $10 \times 10 \mathrm{~mm} 2$ including four circular bonding pads. Chip size can easily be reduced in future design as Fig. 5 shows.

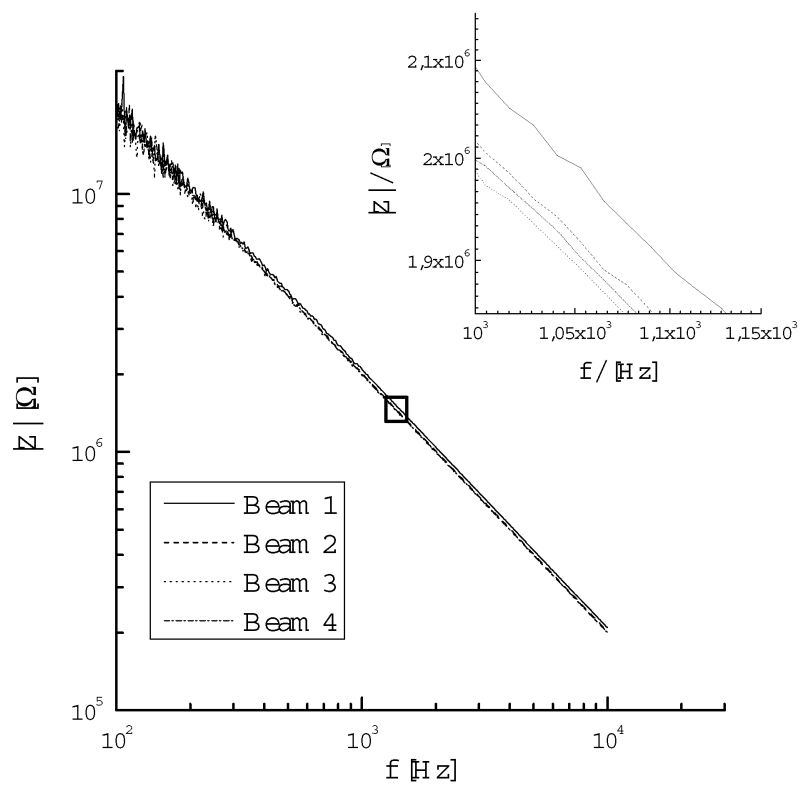

Figure 8. Measured impedances as a function of frequency for four beams on the same accelerometer show a linear relation in a double logaritmic plot. The slope equals -1 proving capacitor like behavior and the capacitance is found to be $79.1 \mathrm{pF}$. 


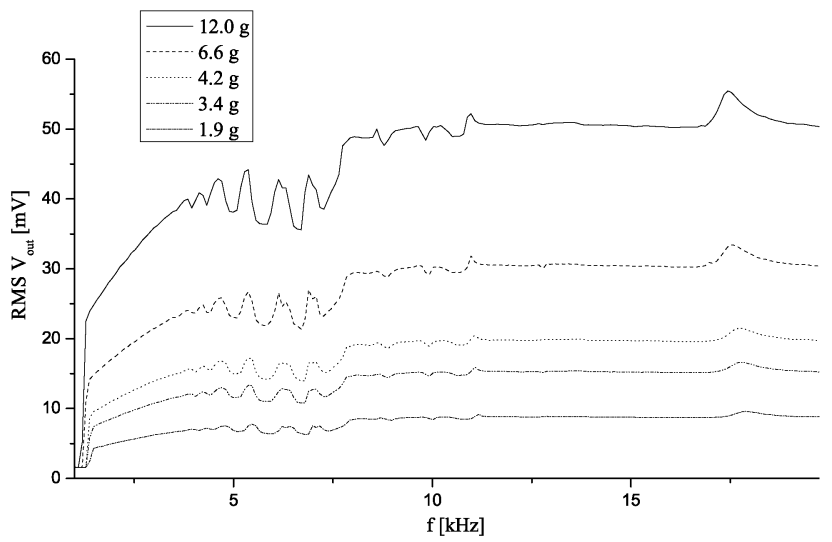

Figure 9. The RMS value of the output voltage is measured for frequencies ranging from $1 \mathrm{kHz}$ to $20 \mathrm{kHz}$. Five different accelerations have been measured ranging from $1.9 \mathrm{~g}$ to $12.0 \mathrm{~g}$. A connstant signal is measure from $10 \mathrm{kHz}$ to the resonance peak at $17.5 \mathrm{kHz}$.

\section{CHARACTERIZATION}

The accelerometer is wire bonded for signal read out (see Fig. 6). The read out signal from the accelerometer is a high impedance signal and a charge amplifier is used to transform the signal into a low impedance signal. The accelerometer can electrically be described as a charge source, a resistor, $\mathrm{R}_{\mathrm{A}}$, and a capacitor, $\mathrm{C}_{\mathrm{A}}$, in parallel (see Fig. 7). The capacitor $C_{F}$ is equal to the capacitance of the accelerometer in order to measure the correct output voltage.

Contrary to the impedance of the resistor the impedance of the capacitor is frequency dependant. At low frequency all current will run through the resistor and the accelerometer will not be operational. The lower end of the bandwidth is determined by this frequency. Fig. 8 shows the measured impedance as a function of frequency ranging from $0.1 \mathrm{kHz}$ to $10 \mathrm{kHz}$. A slope of -1 in a double logarithmic plot proves a capacitor like behavior. The average capacitance for the four beams on the accelerometer is $79.1 \mathrm{pF}$. The slight difference in capacitance is probably due to small difference of the four top electrode areas or local difference in PZT film thickness. The lower end of the bandwidth is therefore proven to be below $0.1 \mathrm{kHz}$.

A shaker (Brüel \& Kjær 4810) setup is used to measure the output as a function of frequency. The acceleration induced by the shaker is frequency dependent up to around $5 \mathrm{kHz}$ and the upper operational frequency of the shaker is $20 \mathrm{kHz}$. Fig. 9 shows the measured RMS value for the output voltage as a function of frequency ranging from 1 $\mathrm{kHz}$ to $20 \mathrm{kHz}$. The frequency sweep is performed for five different accelerations ranging from $1.9 \mathrm{~g}$ to $12.0 \mathrm{~g}$. The acceleration of the vibration is measured with a reference accelerometer. In the range from $5 \mathrm{kHz}$ to $10 \mathrm{kHz}$ an oscillating signal is observed. This is believed to be due to resonance frequencies of the chip holder placed between the shaker and the accelerometer chip. From around $10 \mathrm{kHz}$ to

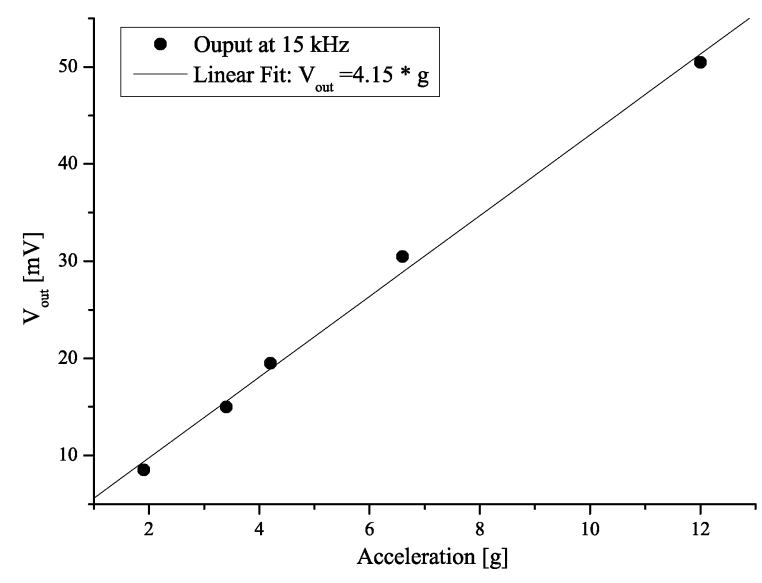

Figure 10. Output signal as a function of acceleration shows a linear relation at $15 \mathrm{kHz}$. The slope of the linear fit equals the sensitivity and is found to be $4.15 \mathrm{mV} / \mathrm{g}$.

the resonance peak at $17.5 \mathrm{kHz}$ the signal is constant for all five accelerations.

Fig. 10 shows the measured RMS values of the output signal in the constant region at $15 \mathrm{kHz}$. Five accelerations ranging from $1.9 \mathrm{~g}$ to $12.0 \mathrm{~g}$ are measured and shows a linear relation. The slope equals the sensitivity and is found to be $4.15 \mathrm{mv} / \mathrm{g}$. This sensitivity is for a single beam where all four beams on the accelerometer coupled in parallel results in a sensitivity of $16.60 \mathrm{mV} / \mathrm{g}$. The capacitance for a single beam is found to be $79.1 \mathrm{pF}$ which results in a charge sensitivity of $0.33 \mathrm{pC} / \mathrm{g}$.

\section{CONCLUSION}

In conclusion, MEMS and TF screen printing technologies have successfully been combined to fabricate a low cost accelerometer with a simple fabrication process only using three lithographic masks and two for the screen printing process. The characterization has shown promising results so far. The sensitivity at $15 \mathrm{kHz}$ is measured to be 4.15 $\mathrm{mV} / \mathrm{g}$ and the resonance peak is found to be at $17.5 \mathrm{kHz}$. A constant sensing region is found from $10 \mathrm{kHz}$ to $16 \mathrm{kHz}$. The lower limit is believed to be much lower but has not yet been proven. The good results motivates for further investigation leading to a tri-axial accelerometer.

\section{ACKNOWLEDGMENT}

The project is a collaboration with InSensor $\mathrm{A} / \mathrm{S}$ and Ferroperm Piezoceramics A/S and is supported by "The Danish National Advanced Technology Foundation".

\section{REFERENCES}

[1] Li-Peng Wang, Richard A. Wolf Jr., Yu Wang, Ken K Deng, Lichun Zou, Robert J. Davis, Susan Troiler-McKinstry, Journal of MEMS, Vol. 12, no. 4, 2003.

$$
0
$$

[2] S. P. Beeby, J. N. Ross, N. M. White; Journal of Micromech Michroeng., Vol. 10, p. 322-328, 2000. 